\title{
An Efficient Imperialism Competitive Algorithm for a Reliable Hub Covering Location Problem
}

\author{
Marjan Sadeghi and Reza Tavakkoli-Moghaddam
}

\begin{abstract}
Hubs are critical elements that benefit networks by switching, distributing and concentrating flows. A hub location problem is common in a lot of industries, such as transportation networks, telecommunication networks, postal delivery networks and express shipment. Furthermore, a transportation network plays a vital role in each country. Nonetheless, during a day many factors such as traffic incidence, natural disasters cause some degradation in the transportation network. As a result, in this paper, we study a reliable hub covering location problem in a degradable transportation network to minimize the total transportation cost. For achieving near-optimal solutions for a real-sized problem an imperialism competitive algorithm (ICA) is proposed. Also the effectiveness of the proposed ICA is shown by some computational experiments. At last, conclusion is provided.
\end{abstract}

Index Terms-Hub location, road, capacity reliability, chance-constraint method, imperialism competitive algorithm.

\section{INTRODUCTION}

Hub location problems (HLPs) have risen when transferring data, passenger, goods and cargo between components of a network became an important issue. Hubs are vital elements, in which benefit networks by switching; distributing and concentrating flows in many aspects such as time budget and economics [1]-[3]. Indeed, hub facilities bring economic scale advantages to a network. Accordingly, a HLP gets high attention in the recent years. Also, this kind of facility location is chiefly common in the transportation network, postal delivery network and express shipment. Alumur and Kara [4] provided a comprehensive survey about HLPs, in which most of the readers are referred to them.

Nowadays, a transportation system is a lifeline of each country, and many other important sectors in a country are influenced by transportation system directly, such as economic and environment. In a day to day, urbanization grows quickly and causes more complexity in the transportation system. As a result, enhancing the transportation system for adaption with daily life in aspect of economic, environmental and efficiency concerns is worthwhile issue [5]. It is influenced by many factors during a day. Unavoidable natural disasters, aging transportation infrastructures, security concerns and occurrence of traffic incidents can be mentioned as degradation factors which affect on the transportation system by road degradation [5].

Manuscript received September 18, 2014; revised November 20, 2014. The authors are with School of Industrial Engineering, Collage of Engineering, University of Tehran, Tehran, Iran (email: marjan_sadeghi@ut.ac.ir, tavakoli@ut.ac.ir).
Roads degradations cause poor performance of the transportation network [6]. Hence, identifying the road's degradations impact on the transportation network can be useful issue in view point of network designer and urban manager.

Degradation factors cause some reduction in road's capacity and some increment in travelling time uncertainty [7]. As a consequence, a transportation network stands in an uncertain situation due to variability of the road's capacity and traveling time. Therefore, a reliable transportation network get high importance in view point of travelers and traffic managers [6]. The definition of reliability in a transportation network is extracted from discipline of reliability engineering [8]. Road capacity reliability is proposed as a probability that assures the incoming flow to a road is less than its available capacity [9], [10].

Since the HLP is commonly applied in transportation systems, and according to elaborated above reasons, this paper tries to investigate the effect and impact of the capacity degradations on the hub networks. This problem is formulated as mixed-integer non-linear mathematical programming (MINLP). Additionally, the objective function tries to minimize the total transportation cost by considering a road capacity reliability constraint. The maximum flow that network can carry is achieved during the optimization.

\section{LITERATURE REVIEW}

The pioneering research about HLPs has been done by O'Kelly [11]. Also the first mathematical formulation is constructed by O'Kelly [12]. Then, Campbell [13] presented a first linearization of O'Kelly [12] model. After that, the majority papers in this scope focus on linearization of O'Kelly's model [12] and solving procedure to minimize total transportation cost (e.g., [14]-[18]). Many different types of HLP were introduced by Campbell [14], such as $p$ hub median, $p$-hub center and $p$-hub covering. For more detailed information in this field [19], [20] are comprehensive review, in which most of the readers are referred to them.

The concept of reliability in degradable transportation network was introduced as connectivity reliability, in which it is a probability assures entire nodes in a network remain connected [21]. After that Asakura and Kashiwadani [22] presented time reliability definition as probability ensures travelers arrive to the destination successfully, in a specific interval. The first framework for analyzing degradable networks has been constructed by Du and Nicholson [23]. Then, capacity reliability was provided by Chen et al. [24] and [25] as explained in Section I. Dianhai et al. [26] presented a comprehensive survey about different kind of 
reliability in transportation network.

\section{MATHEMATICAL MODEL}

In this research, a transportation network is assumed as a graph $G(N, A)$, where $N$ and $A$ indicate to set of nodes and set of links, respectively. The transportation network is configured by set of links as roads. Also, capacity of each link $i \rightarrow k \in A$ is considered as random variable according to stochastic degradations. The objective function tries to minimize the transportation cost. Furthermore, the maximum flows, which can be carried in the network, are determined by considering a capacity reliability constraint, based on road degradations. A chance-constraint method is used to calculate the capacity reliability constraint. The number of hubs is determined as $P$ in advance. Non-hub nodes are allocated to only one hub facility, single allocation. Necessary notations are provided as follows.

\section{Sets:}
$i, j \in N$
Set of nodes.
$k, m \in N$
Set of hubs.

\section{Parameters:}

$\begin{array}{ll}\mu & \text { Discount multiplier between hubs. } \\ P & \text { Number of hubs. } \\ M & \text { A large fixed number. } \\ f_{k} & \text { Fixed setup cost for hub } k . \\ r_{k} & \text { Covering radius of hub } k . \\ \alpha_{i k} & \text { Road capacity exceedance probability for } \\ & \text { link } i \rightarrow k . \\ \theta_{i k} & \text { Degradable fraction of link } i \rightarrow k . \\ C_{i k} & \text { Link capacity for link } i \rightarrow k . \\ \bar{c}_{i k} & \text { Designed capacity for link } i \rightarrow k . \\ \mathrm{w}_{i j} & \text { Total emanated flows between node } i \text { and node } \\ & j . \\ \mathrm{Co}_{i k} & \text { Transportation cost unit between link } i \rightarrow k . \\ \mathrm{Co}_{i j k m} & \text { Total transportation cost for route } i \rightarrow k \rightarrow \\ & m \rightarrow j .\end{array}$

\section{Variables:}

$X_{i k} \quad$ Flows which emanating from node $i$ to hub $k$.

$X_{i j k m} \quad$ Is equal to 1 if emanated flow from $i$ to $j$ using hubs $k$ and $m$; otherwise, it is equal to 0 .

$Z_{i k} \quad$ Is equal to 1 if node $i$ is allocated to hub $k$; otherwise, it is equal to zero.

where the transportation cost in route $i \rightarrow k \rightarrow m \rightarrow j$ is calculated as (1):

$$
\mathrm{Co}_{i j k m}=\mathrm{Co}_{i k}+\mu \mathrm{Co}_{k m}+\mathrm{Co}_{m j}
$$

For achieving the road capacity reliability constraint by a chance-constraint method a certain probability is considered as road exceedance probability. This is a probability defined for situation that flows on the road be greater than road's designed capacity, in which is shown by $\alpha_{i k}$ for link $i \rightarrow k$. Eq. (2) represents a mathematically form of exceedance probability definition:

$$
P\left\{\mathrm{X}_{i k} \geq C_{i k}\right\}=P\left\{C_{i k} \leq \mathrm{X}_{i k}\right\} \leq \alpha_{i k}
$$

The left hand side of (2) can be reformulated as (3) by considering cumulative distribution function (CDF) definition:

$$
F_{C_{i k}}\left(\mathrm{X}_{i k}\right)=P\left\{C_{i k} \leq \mathrm{X}_{i k}\right\}
$$

Eq. (4) is achieved based on (2) and (3):

$$
F_{C_{i k}}\left(\mathrm{X}_{i k}\right) \leq \alpha_{i k}
$$

Eq. (4) can be reversed as (5), because CDFs are monotonic one-to-one:

$$
\mathrm{X}_{i k} \leq F_{C_{i k}}^{-1}\left(\alpha_{i k}\right)
$$

In this paper, for determining Constraint (5), the road capacity is considered as uniform random variable. Also, the designed capacity and worst-degraded capacity are assumed as upper and lower bounds of the uniform distribution, respectively. Furthermore, the worst-degraded capacity for link $i \rightarrow k$ is calculated by a fraction $\theta_{i k}$ of the designed capacity, namely degradable fraction. As a result, (6) shows the inverse $\mathrm{CDF}$ for uniform random variable $C_{i k}$ :

$$
\begin{aligned}
F_{C_{i k}}^{-1}\left(\alpha_{i k}\right)=c_{i k}= & \theta_{i k} \bar{c}_{i k}+\alpha_{i k} \bar{c}_{i k}\left(1-\theta_{i k}\right) \\
& =\bar{c}_{i k}\left[\theta_{i k}\left(1-\alpha_{i k}\right)+\alpha_{i k}\right]
\end{aligned}
$$

where $c_{i k}$ is an instance of the uniform random variable $C_{i k}$. It is remarkable that $\bar{c}_{i k}$, the designed capacity for link $i \rightarrow k$, has a deterministic value. Finally, the road capacity reliability constraint is obtained by (7):

$$
\mathrm{X}_{i k} \leq \bar{c}_{i k}\left[\theta_{i k}\left(1-\alpha_{i k}\right)+\alpha_{i k}\right]
$$

Mathematical Formulation:

$$
\begin{aligned}
& \text { Min } Z \\
& =\sum_{k \in N} f_{k} Z_{k k} \\
& +\sum_{i \in N} \sum_{j \in N} \sum_{k \in N} \sum_{m \in N} W_{i j} C o_{i j k m} X_{i j k m} \\
& \text { s.t }
\end{aligned}
$$

$$
\begin{aligned}
& \sum_{k \in N} Z_{i k}=1 \quad \forall i \in N \\
& \sum_{k \in N} Z_{k k}=p \\
& \sum_{k \in N} \sum_{m \in N} X_{i j k m}=1 \quad \forall i, j \in N \\
& Z_{i k} \leq Z_{k k} \quad \forall i, k \in N \\
& Z_{i k}+Z_{j m}-\leq X_{i j k m} \quad \forall i, k, m, j \in N \\
& \mathrm{X}_{i k} \leq \bar{c}_{i k}\left[\theta_{i k}\left(1-\alpha_{i k}\right) \quad \forall \quad+\alpha_{i k}\right] \quad \forall i, k \in N \\
& \mathrm{X}_{k m} \leq \bar{c}_{k m}\left[\theta_{k m}\left(1-\alpha_{k m}\right) \quad \forall k, \alpha_{k m}\right] \quad \forall k, m \in N \\
& C o_{i k} Z_{i k} \leq r_{k} Z_{k k} \quad \forall i, k \in N \\
& X_{i j k m}=\{0,1\}, Z_{i k}=\{0,1\}, \mathrm{X}_{i k} \geq 0
\end{aligned}
$$

The objective function (8) is to minimize the 
transportation cost consisting of the transportation cost and hubs fixed establishment cost. Constraint (9) requires single allocation. Constraint (10) ensures the number of hubs is predetermined, which is shown by $P$. Constraint (11) states that emanated flows between each O-D pair can be served by two hubs. Constraint (12) indicates only after establishing a hub nodes, non-hub nodes can be allocated to it. Constraint (13) assures that flows can be served by hubs $k$ and $m$ when nodes $i$ and $j$ are allocated to them, respectively. Constraints (14) and (15) are road capacity reliability constraints, between non-hub nodes and hubs; and between hubs, respectively. The covering constraint is shown by Constraint (16) based on the transportation cost. At last, the decision variables are shown by constraint (17).

The emanated flows between non-hub nodes and hub nodes, and between hub nodes are calculated by (18) and (19), respectively:

$$
\begin{aligned}
\sum_{m \in N} \sum_{j \in N} W_{i j} \mathrm{X}_{i j k m} & +\sum_{m \in N} \sum_{j \in N} W_{j i} \mathrm{X}_{j i m k} \\
& =\mathrm{X}_{i k} \quad \forall i, k \in N \\
\sum_{i \in N} \sum_{j \in N} W_{i j} \mathrm{X}_{i j k m} & +\sum_{i \in N} \sum_{j \in N} W_{j i} \mathrm{X}_{j i m k} \\
& =\mathrm{X}_{m k} \quad \forall m, k \in N
\end{aligned}
$$

\section{SOLUTION APPROACH}

The validity of the presented model is tested by GAMS 22.9 software. For small-sized problems, we successfully achieve to an optimal solution by using a branch-and-reduce approach in the BARON solver up to 10 nodes. Nonetheless, HLPs are well-known as NP-hard problem [4], and the presented model is MINLP model. Consequently, the obtaining optimal solution especially for real-sized problem is computationally hard. As a result, we propose an ICA for obtaining near-optimal solutions in reasonably time for a real-sized problem.

\section{A. Proposed ICA}

The ICA is a population based evolutionary algorithm which is introduced by Atashpaz-Gargari and Lucas [27] based on socio-political human evolution. The initial populations are well-known as country. During the optimization procedure, countries are divided to imperialisms and colonies, based on objective function. The most powerful imperialism catches more colonies than the other imperialisms. Indeed, colonies are divided between imperialisms according their power, which is calculated by an objective function. Then, colonies start to move toward their imperialism, which is well-known as assimilation. In fact, assimilation provides competent exploitation for the algorithm. In this paper, we add a crossover operator in the ICA to have better sharing information between colonies [28].To have a better exploration in the ICA, the revolution operator is performed, in which colonies move randomly to find the better place. Both assimilation and revolution provide an inner empire competition. On the other hand, for the enter empire competition the weakest imperialism is determined, then the weakest colonies of this imperialism is considered and divided between the others imperialism, randomly. If there is imperialism without any colony, it collapses and is divided between the other imperialism. More detailed information about the ICA is explained comprehensively by Mohammadi et al. [28].

\section{B. Solution Representation}

Solution representation is the most important part of designing a meta-heuristic. In this paper, we use $1 \times(n+$ $p)$ matrix as continuous solution encoding (CSE), in which $n$ and $p$ indicate to number of nodes and hubs, respectively. The matrix is divided to two part as allocation and location phases. Bit 1 up to bit $n$ configure the allocation phase and the others make the location phase. The bits of the allocation part are filled by randomly generated data, between 0 and 1 . Then, they are sorted by descending order. Also, location part is filled by randomly generated numbered between 1 and 9; by ascending order, it is notable the last bit, $(n+p)$, is equal to the number of nodes. The bits of a location part show the hubs, and in allocation part each non-hub node is allocated to its right hub. Fig. 1 illustrates the explained solution representation for 10 nodes and 4 hubs. In the location part, nodes 3, 5, 8 and 2 are determined as hubs, and in allocation part nodes $9,1,7,10$, 4 and 6 are allocated to 3,3, 5, 8, 2 and 2, respectively.

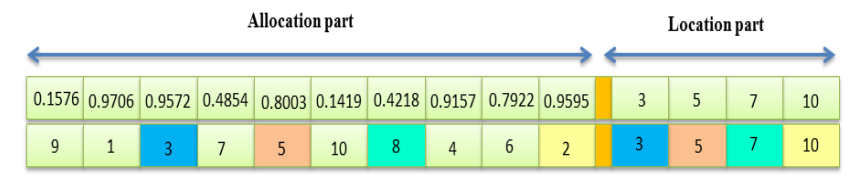

Fig. 1. Solution representation for 10 nodes.

\section{EXPERIMENTAL RESULTS}

The validity of the proposed ICA is examined with comparing results between the proposed ICA and GAMS software for small-sized and randomly generated test problems. The obtained results of objective functions from GAMS and ICA and related gaps for small-sized problems are tabulated in Table I. Also, the objective function for large-sized problems is calculated and embedded in this table. Furthermore, we use a number of nodes\# number of hubs notation for more simplicity.

TABLE I: COMPUTATIONAL COMPARISON BETWEEN THE GAMS AND PROPOSED ICA

\begin{tabular}{c|c|c|c}
\hline $\begin{array}{c}\text { nodes\# } \\
\text { hubs }\end{array}$ & OFV of GAMS & OFV of ICA & Gap (\%) \\
\hline $5 \# 3$ & 692.3 & 692.3 & 0.0 \\
$7 \# 4$ & 985.840 & 985.840 & 0.0 \\
$8 \# 4$ & 114.380 & 114.380 & 0.0 \\
$9 \# 5$ & $1,376.850$ & $1,378.999$ & 0.1 \\
$10 \# 5$ & $1,499.229$ & $1,515.9$ & 1.09 \\
$10 \# 7$ & $1,907.951$ & $1,922.23$ & 0.704 \\
$20 \# 12$ & ----- & $4,657.951$ & ---- \\
$30 \# 15$ & ---- & $7,157.0$ & ----- \\
$40 \# 15$ & ----- & $9,000.002$ & ---- \\
$50 \# 25$ & ----- & $12,116.971$ & ---- \\
\hline
\end{tabular}

The gap between GAMS software and the proposed ICA is calculated by: 


$$
\left(O F V_{I C A}-O F V_{G A M S} / O F V_{I C A}\right) \times 100
$$

\section{CONCLUSION}

In this paper, we have presented a new mathematical modeling for a hub location problem in a degradable transportation network. The road capacity has been considered as a uniform random variable. Also, to achieve the capacity reliability constraint a chance-constraint method has been used, by considering a specific road capacity exceedance probability. The validity of the presented mathematical model has been tested by the GAMS solver. Furthermore, for solve the problem for small-sized instances up to 10 nodes, the GAMS solver has been used successfully. However, to achieve the nearoptimal solutions for real-sized problems, we have proposed an ICA. The effectiveness of the proposed ICA has been compared with achieved results from the GAMS software, for small-sized problems. Also, we have obtained nearoptimal solutions for real-sized problems up to 50 nodes successfully.

\section{REFERENCES}

[1] I. Contreras, E. Fernandez, and A. Marin, "Tight bounds from a path based formulation for the tree of hub location problem," Computers \& Operations Research, vol. 36, pp. 3117-3127, 2009.

[2] H. A. Eiselt and V. Marianov, "A conditional p-hub location problem with attraction functions," Computers \& Operations Research, vol. 36 pp. 3128-3135, 2009.

[3] M. R. Silva and C. Cunha, "New simple and efficient heuristics for the uncapacitated single allocation hub location problem," Computers \& Operations Research, vol. 36, pp. 3152-3165, 2009.

[4] S. Alumur and B. Y. Kara, "Network hub location problems: The state of the art," European Journal of Operational Research, vol. 190, no. 1, pp. 1-21, 2008.

[5] H. K. Lo, X. W. Luo, and B. W. Y. Siu, "Degradable transport network: Travel time budget of travelers with heterogeneous risk aversion," Transportation Research - Part B, vol. 40, no. 9, pp. 792806, 2006.

[6] W. H. Dianhai, Q. I. Sheng, and X. U. Cheng, "Reviewing traffic reliability research," Journal of Transportation Systems Engineering and Information Technology, vol. 10, no. 5, pp. 12-21, 2010.

[7] H. K. Lo and Y. K. Tung, "Network with degradable links: Capacity analysis and design," Transportation Research Part B, vol. 37, no. 4, pp. 345-363, 2003.

[8] G. H. Sandler, System Reliability Engineering, Englewood Cliffs, New Jersey: Prentice-Hall, 1963.

[9] A. Chen, H. Yang, and H. K. Lo, "A capacity related reliability for transportation network," Journal of Advanced Transportation, vol. 33, no. 2, pp.183-200, 1999.

[10] A. Chen, H. Yang, and H. K. Lo, "Capacity reliability of a road network: An assessment methodology and numerical results," Transportation Research Part B, vol. 36, no. 3, pp. 225-252, 2002.

[11] M. E. O'Kelly, "The location of interacting hub facilities," Transportation Science, vol. 20, no. 2, pp. 92-106, 1986.

[12] M. E. O'Kelly, "A quadratic integer program for the location of interacting hub facilities," European Journal of Operational Research, vol. 32, pp. 393-404, 1987.

[13] J. F. Campbell, "Integer programming formulations of discrete hub location problems," European Journal of Operational Research, vol. 72, no. 2,pp. 387-405, 1994.

[14] J. F. Campbell, "Hub location and the p-hub median problem," Journal of Operations Research, vol. 44, no. 6, pp. 1-13, 1996.

[15] A. T. Ernst and M. Krishnamoorthy, "Efficient algorithms for the uncapacitated single allocation p-hub median problem," Location Science, vol. 4, no. 3, pp. 139-154, 1996.

[16] M. E. O’Kelly, D. Skorin-Kapov, and J. Skorin-Kapov, "Lower bounds for the hub location problem," Management Science, vol. 41, no.4, pp. 713-721, 1995.
[17] D. Skorin-Kapov, J. Skorin-Kapov, and M. E. O’Kelly, “Tight linear programming relaxations of uncapacitated p-hub median problems," European Journal of Operational Research, vol. 94, no. 3, pp. 582593, 1996.

[18] B. Y. Kara and B. C. Tansel, "On the single-assignment p-hub center problem," European Journal of Operational Research, vol. 125, pp. 648-655, 2000.

[19] J. F. Campbell and M. E. O'Kelly, "Twenty-five years of hub location research," Journal of Transportation Science, vol. 46, no. 2, pp. 153169, 2012

[20] R. Z. Farahani, M. Hekmatfar, A. B. Arabani, and E. Nikbakhsh, "Hub location problems: A review of models, classification, techniques and application," Computers \& Industrial Engineering, vol. 64, pp. 1096-1109, 2013.

[21] Y. Iida and H. Wakabayashi, "An approximation method of terminal reliability of a road network using partial minimal path and cut set," in Proc. the $5^{\text {th }}$ WCTR, Yokohama, 1989, pp. 367-380.

[22] Y. Asakura and M. Kashiwadani, "Road network reliability caused by daily fluctuation of traffic flow," in Proc. the $19^{\text {th }}$ PTRC Summer Annual Meeting, Brighton, 1991, pp. 73-84.

[23] Z. P. Du and A. Nicholson, "Degradable transportation systems: Sensitivity and reliability analysis," Transportation Research Part B, vol. 31, pp. 225-237, 1997.

[24] A. Chen, H. Yang, and H. K. Lo, "A capacity related reliability for transportation network," Journal of Advanced Transportation, vol. 33, no. 2, pp. 183-200, 1999.

[25] A. Chen, H. Yang, and H. K. Lo, "Capacity reliability of a road network: An assessment methodology and numerical results," Transportation Research Part B, vol. 36, no. 3, pp. 225-252, 2002.

[26] W. H. Dianhai, Q. I. Sheng, and X. U. Cheng, "Reviewing traffic reliability research," Journal of Transportation Systems Engineering and Information Technology, vol. 10, no. 5, pp.12-21, 2010.

[27] E. Atashpaz-Gargari, F. Hashemzadeh, R. Rajabioun, and C. Lucas, "Colonial competitive algorithm, a novel approach for PID controller design in MIMO distillation column process," International Journal of Intelligent Computing and Cybernetics, vol. 1, no. 3, pp. 337-355, 2008.

[28] M. Mohammadi, F. Jolai, and R. Tavakkoli-Moghaddam, "Solving a new stochastic multi-mode p-hub covering location problem considering risk by a novel multi-objective algorithm," Applied Mathematical Modeling, vol. 37, no. 24, pp. 10053-10073, 2013.

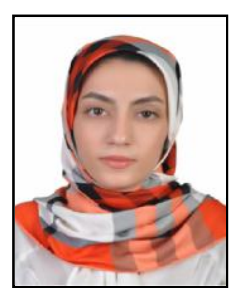

Marjan Sadeghi received her M.Sc. degree in industrial engineering from College of Engineering at University of Tehran in Iran in 2014. She obtained her B.Sc. degree in applied mathematics from the Khajeh-Nasir Toosi University of Technology in Tehran, Iran in 2011. Her research interests include hub location, transportation, operation research, meta-heuristic methods, exact optimization methods. Marjan Sadeghi has submitted four papers related to hub location problems to well-known ISI journals.

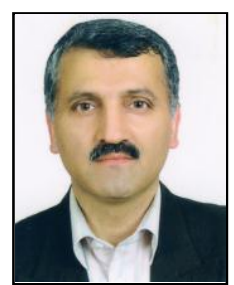

Reza Tavakkoli-Moghaddam is a professor of industrial engineering at College of Engineering, University of Tehran in Iran. He obtained his Ph.D. in industrial engineering from the Swinburne University of Technology in Melbourne in 1998, his M.Sc. degree in industrial engineering from the University of Melbourne in Melbourne in 1994 and his B.Sc. in industrial engineering from the Iran University of Science and Technology in Tehran in 1989. His research interests include facility layouts and location design, cellular manufacturing systems, sequencing and scheduling, and using meta-heuristics for combinatorial optimization problems. He serves as a member of editorial boards of the International Journal of Engineering and Iranian Journal of Operations Research as well as three other Iranian journals. He is the recipient of the 2009 and 2011 Distinguished Researcher Award and the 2010 Distinguished Applied Research Award at the University of Tehran, Iran. He has been selected as a national iranian distinguished researcher for 2008 and 2010. Professor TavakkoliMoghaddam has published 4 books, 15 book chapters and more than 500 papers in reputable academic journals and conferences. 